(C2016 IEEE. Reprinted, with permission, from A. Alanazi, and K.M. Elleithy, "QKDP's comparison based upon quantum cryptography rules.” In Proceedings of 2016 Second International Conference on Mobile and Secure Services (MobiSecServ), Gainesville, FL, 2016. DOI: 10.1109/MOBISECSERV.2016.7440227.

This material is posted here with permission of the IEEE. Such permission of the IEEE does not in any way imply IEEE endorsement of any of the University of Bridgeport's products or services. Internal or personal use of this material is permitted. However, permission to reprint/republish this material for advertising or promotional purposes or for creating new collective works for resale or redistribution must be obtained from the IEEE by writing to pubs-permissions@ieee.org. By choosing to view this document, you agree to all provisions of the copyright laws protecting it. 


\title{
Optimized Node Selection Process for Quality of Service Provisioning over Wireless Multimedia Sensor Networks
}

\author{
Adwan Alanazi and Khaled Elleithy \\ Department of Computer Science and Engineering \\ University of Bridgeport, CT-06604, USA \\ aalanazi@my.bridgeport.edu; elleithy@bridgeport.edu
}

\begin{abstract}
Several quality of service (QoS) routing strategies focus on the improvement of throughput and end-to-end delays in wireless sensor networks (WSNs). With emergence of wireless multimedia sensor networks (WMSNs), data traffic can be poised into reliability-demanding data packets and time-sensitive data packets. In such situations, node optimization and load balancing can improve QoS provisioning. Thus, the trade-off between network lifetime and ensuring the QoS provisioning has been of paramount importance. This paper introduces the Optimized Node Selection Process (ONSP) approach for robust multipath QoS routing for WMSNs. This approach is based on determining the optimized node that helps resilient route discovery for improving the QoS parameters. The selection of optimized nodes make the solid chain for route selection using residual energy and received signal strength indicator (RSSI). The second goal of this approach is to prolong the network lifetime by introducing the load-balancing algorithm, which determines the optimized and braided paths. These paths avoid bottlenecks and improves throughput, end-to-end delay, on-time packet delivery and prolongs the network lifetime.
\end{abstract}

Keywords - Wireless sensor networks, Quality of service, Routing, optimized path, braided paths.

\section{INTRODUCTION}

Wireless sensor networks (WSNs) comprise of the promising technology mounted for resolving several solutions, covering military, health, civilian, commercial and environmental applications [1],[2],[3],[4],[5]. WSNs involve a large number of small and low-cost sensors, which are equipped with computation capabilities and wireless communication [6]. However, despite the benefits that the exploitation of a WSN brings, their deployment is limited due to energy limitations posed by the sensor nodes. The energy expenditure of wireless sensor networks depends on the data processing, environmental sensing and wireless communication. Hence, most of the QoS routing protocols aim mostly at the accomplishment of the energy preservation. Since some of the routing protocols designed for WSNs follow the attainment of energy efficiency, they are practically unsuitable for QoS provision in WSNs[7].

The latest advances in complementary metal-oxide semiconductor (CMOS) cameras and small microphones have led to the development of Wireless Multimedia Sensor Networks (WMSN) as a class of wireless sensor networks.
WMSN is a network of wirelessly interconnected sensor nodes that are able to extract multimedia content such as images, audio and video about the ambience and send that to the sink. Routing protocols in WMSNs should be designed with minimum communication overhead and low-processing convolution. The sensor nodes generally function in pervasive locations without user involvement. Thus, the routing should be done by using a load-balancing scheme to take adaptive decisions for balancing the load for each route with respect to the external environment. Furthermore, the routing protocols must be performance-efficient and scalable [8]. In wireless multimedia sensor networks, it is important to deploy the powerful load-balancing routing approaches to support applications such as security monitoring, battlefield intelligence, environmental tracking and emergency response [9]. These applications require multipath QoS routing protocols to create the tradeoff between energy consumption and QoS parameters prior to delivering the data to the sink node [10],[11]. The multi-path QoS routing protocols establish multiple paths to balance the network traffic between sourcenode to destination-node. In literature, there are many approaches available for conventional networks. However, these approaches are too complicated to be considered WMSNs. In Addition, WMSNs differ in nature from wired network because nodes in WMSN hold a single queue that is connected with a single transmitter [9],[12]. The main purpose of introducing the multi-path routing protocol is fault tolerance, bandwidth aggregation, reducing delay and load balancing [13]. We focus on the multi-path QoS routing protocol for improving the network lifetime and improving the throughput, reducing the end-to-end delay as well as on-time packet delivery.

In this paper, we propose Optimized Node Selection Process: ONSP is an energy efficient and quality of service based multi-path routing protocol for wireless multimedia sensor networks that selects optimized disjoint and braided paths to achieve load balancing though splitting the network traffic on the primary path (optimized path) and braided paths (other alternative paths). Optimized node selection process improves the delivery of data reliability using a received signal strength indicator and residual energy models. In order to transmit the data over optimized and braided paths, the load-balancing algorithm is used to guarantee load-balancing 
over the network traffic to avoid the congestion and improves the throughput while reducing latency.

The remnants of the paper are organized as follow: In Section 2, we present the Optimized Node Selection Process. Section 3 , describes the load-balancing algorithm. Section 4, presents simulation-setup and performance evaluation. Finally, section 5 concludes the paper.

\section{OPTIMIZED NODE SELECTION PROCESS}

The optimized node selection process involves the use of mathematical formulation to determine the node having enough resources to forward the data to next node based on residual energy, optimal path and received signal strength indicator (RSSI).

\section{A. RESIDUAL ENERGY AND OPTIMAL PATH}

To determining the optimum node discovery, each path between source node and destination node is defined as $P=\left(P_{1}, P_{2}, \ldots, P_{n}\right)$. Where, $\mathrm{P}$ : set of paths, $P_{1}$ : the source node and $P_{n}$ : the base station, which spans over $P_{n}-2$ (intermediate nodes between source and destination). Thus, residual energy of each intermediate node can be determined after creating the corresponding path and finishing the one event-detection cycle obtained as follows:

$$
R_{e}=P+\sum_{I=1}^{n-1} E\left(P_{i}, P_{i+1}\right)
$$

where ' $R_{e}$ ' is the residual energy of each intermediate node on the path. The residual energy of each node could be different and depending on the participation of the communication node and how much node involves in the communication and $E\left(P_{i}, P_{i+1}\right)$ is the required energy for routing the message between two intermediate nodes $P_{i}$ and $P_{i+1}$. Let us assume ' $\mathrm{X}$ ' is the set of possible paths $X=x_{1}, x_{2}, x_{3}, \ldots, x_{n}$ between source node and destination. Therefore, optimum path between two nodes can be determined as

$$
x_{k}=\max \left\{\left(R_{e}\right): x_{k} \in X\right.
$$

where ' $x_{k}$ ' is the optimum path between two nodes.

\section{B. RECEIVED-SIGNAL STRENGTH INDICATOR (RSSI)}

The signal strength is translated into distance. As a result, the existing techniques experience the problem due to noise interference, multi-path fading, and irregular signal propagation that highly affect the correctness of ranging estimate. To overcome these problems, we apply an improved approach of determining the RSSI for optimized routing path. The localization accuracy can be endorsed to fulfill the requirements for optimization. We apply localization refinement, region partition and regular node placement. In RSSI, the distance between transmitter ' $T_{x}$ ' and receiver ' $R_{x}{ }^{\prime}$ can be obtained by using log-normal shadowing approach described as:

$$
R_{p}(r)=R_{p}(r)-10 n \log \left(\frac{r}{r}\right)+G^{\circ}
$$

where $R_{p}(r)$ : the received power, $R_{p}(r)$ : received power of point, $r$ : the distance between receiver and transmitter, $r$ : reference distance, $n$ : exponent factor for power loss, and $G^{\circ}$ :Gaussian random variable that is used for the change of the power when setting the distance. In practices, basic shadowing model is used for determining the distance based on RSSI.

$$
R_{p}(r)=R_{p}(r)-10 n \log \left(\frac{r}{r}\right)
$$

We assume that reference distance is 1 meter, so we can obtain resilient RSSI as follows:

$$
R S S I=R_{p}(r)=\Delta \forall-10 \text { nlogr }
$$

where $\Delta \forall:$ received signal strength. This RSSI-based localization covers mentioned limitations and also helps determine an optimized route.

\section{DYNAMIC LOAD BALANCING}

To balance the load over the network, the traffic is routed through multiple routes. We use the dynamic load-balancing approach for all paths from source to destination. The bandwidth is distributed over these paths according to the traffic load. The paths consist of optimized and braided paths. The Optimized path is the primary path that is allotted more Bandwidth and braided paths are alternate paths to balance the traffic depicted in Figure 1. The bandwidth is reserved for each route based on optimized load balancing (OLB) algorithm. Let us assume that expected load " on optimized and braided paths need to be updated. This is the reason that original " is distributed on the all candidate paths and their respective values are updated as follows

$$
\begin{gathered}
=-\forall(\vartheta, \varpi) \Phi+\Phi \quad \forall \in R_{\Phi} \\
=-\forall(\vartheta, \varpi) \Phi+(K-) \quad \forall \in R_{\Phi 1} \\
=-\forall(\vartheta, \varpi) \Phi \quad \forall \notin\left(R_{\Phi} \cup R_{\Phi 1}\right)
\end{gathered}
$$

where ' $\vartheta$ ': source, and ' $\varpi$ ': distination. We deduct the bandwidth-demand value ${ }^{\prime} \forall(\vartheta, \varpi) \Phi^{\prime}$ that is passed through each link. Each link creates optimized ' $R_{\Phi}^{\prime}$ and braided paths ${ }^{\prime} R_{\Phi 1}^{\prime}$ over the network. Optimized path ' $R_{\Phi}^{\prime}$ is the primary route. The tangible reservation is ${ }^{\prime} \Phi^{\prime}$. In case of reserved bandwidth for optimized load balancing, $\Phi=\Phi$ for all the links $L_{1} \in R_{\Phi}$. From another perspective, in case of reserved bandwidth-delay for OLB, we divide an end-to-end delay into different link delay limitations. As a result, each link along the optimized and braided paths has the different reserved bandwidth' $\Phi^{\prime}$. 
Thus, $\Phi \geq \Phi$. For the links along a braided path ' $R_{\Phi 1}$ ', the $(K-)$ in both cases ranges between 1 and ' $\Phi$ ' based on the shared bandwidth on the links ' $L_{1}$ '. $K$ : Initial energy of link and : residual energy of link, which are calculated before and after reserving the bandwidth for paths of the network. The expected load" for each path is updated over each link. In the end, having setup the all possible routes, the most utilized links will get highest value.

Algorithm 1: Determining the optimized and braided path for end-to-end bound delay ' $ב$ 'and bandwidth of $\Phi$.

1. Input: Optimized specification $(\vartheta, \varpi, \Phi, \beth)$

2. Expected load of each link, residual energy of link, and total energy $\Sigma$

3. Set $\vartheta$ of $\mathrm{T}$ candidate pair of braided pairs $\left(M_{1}, M_{2}\right)$

4. Output: optimized path $R_{\Phi}$ and braided path $R_{\Phi 1}$

5. While all links $L_{1}$ do

6. $t_{\Delta}=\frac{-}{K}$

7. end while

8. $O_{\min }=$ infinity $; O_{\min }$ : $($ Rest of links except optimized and braided links)

9. while each braided pairs $(\mathrm{M}, \mathrm{N}) \in \vartheta$ that meets the requirements of $(\beth, \Phi)$ do

10. Divide $ב$ individually along the braided pairs $M_{1}$ and $\mathrm{M}_{2}$

11. Recalculate the residual energy of link

12. Recalculate the link costs $t_{\Delta}=\frac{-}{K}$

13. Recalculate the network metric $N_{m}$

14. If $N_{m}<O_{\min }$ then

15. $O_{\min }=N_{m} ; R_{\Phi}=M_{1} ; R_{\Phi 1}=M_{2}$

16. endif

17. End while

18. If $O_{\min }>\delta ; \delta$ : value of braided link

19. Reject $O_{\min }$

20. else

21. Choose $R_{\Phi}, R_{\Phi 1}$ as optimized and braided links for routing.

22. end if

\section{SIMULATION SETUP}

In order to analyze the performance of the optimized node selection process for path discovery. We observed a wireless multimedia sensor network that was constituted the size $400 \mathrm{~m}$ $\mathrm{x} 400 \mathrm{~m}$. The performance of ONSP is compared with other QoS routing protocols: Multi-Path and Multi-SPEED (MMSPEED) Protocol, Multimedia Geographic Routing (MGR) and Sequential Assignment Routing (SAR). The rationale behind selecting these protocols is that they are probabilistic protocols and focusing on improving the QoS. Our proposed protocol is having same features. Thus, comparison would help determine effectiveness of our protocol. Thus, the network topology considered the following metrics:

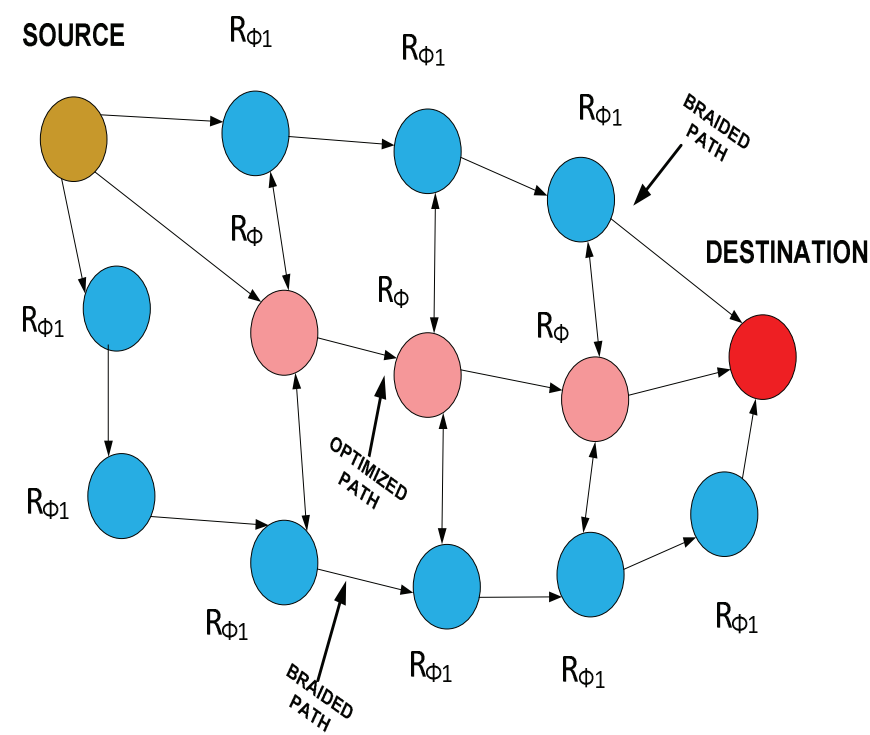

Figure 1: Optimized route discovery process using load-balancing approach

- A static sink is set farther from the sensing field.

- Each node is initially allotted uniform energy.

- $\quad$ Each node senses the field at the variable rates and is responsible for forwarding the data to sink node.

- The sensor nodes are 50\% mobile.

- $\quad$ Each sensor node possesses homogenous capabilities involving the same communication capacity and computing resources.

- The location of sensor nodes is determined in advance.

The aforesaid network topology is suitable for several WMSNs applications, such as home monitoring, and reconnaissance, airport surveillance, biomedical applications, home automation, fire detection, agriculture and machine failure diagnosis. In the actual application of a proposed approach, it may be used for airport surveillance where the sensor nodes are static and mobile, which are used for monitoring the travelers and staff members. The simulation was conducted by using network simulator-2[14]. The scenario consists of 500 homogenous sensor nodes with initial energy of 4 joules. The base station is located at point $(0,500)$. The size of the packets is 256 bytes. The residual energy of each node after 7 cycles is calculated based on the residual energy model described in section 2 . The rest of parameters are explained in Table 1.

Based on simulation, we are interested in the following metrics.

- Average delivery rate

- Average energy consumption

- End-to-end delay

- Lifetime

A. Average delivery rate 
Table 1: Simulation parameters and its corresponding values

\begin{tabular}{|l|l|}
\hline PARAMTERS & \multicolumn{1}{|c|}{ VALUE } \\
\hline Size of network & $400 \times 400$ square meters \\
\hline Number of nodes & 500 \\
\hline Queue-Capacity & 25 Packets \\
\hline Mobility Model & Random way mobility model \\
\hline $\begin{array}{l}\text { Maximum number of } \\
\text { retransmissions allowed }\end{array}$ & 03 \\
\hline Initial energy of node & 4 joules \\
\hline Size of Packets & 256 bytes \\
\hline Data Rate & 250 kilobytes/second \\
\hline Sensing Range of node & 40 meters \\
\hline Simulation time & 9 minutes \\
\hline Average Simulation Run & 08 \\
\hline Base station location & $(0,500)$ \\
\hline Transmitter Power & 12 mW \\
\hline Receiver Power & 13 mW \\
\hline Buffer threshold & 1024 Bytes \\
\hline
\end{tabular}

One of the important metrics in investigating the routing protocols is an average delivery ratio. In Figure 2, node failure probability and an average delivery ratio are depicted. ONSP outperforms other routing protocols: MMSPEED, MGR and SAR. The average delivery ratio decreases by node failure. However, node failure highly affects other participant routing protocols as compared with ONSP. The reason of the better performance of ONSP is the inclusion the load-balancing algorithm and optimized node processing approach based on residual energy and RSSI. The performance of ONSP reduces maximum to $18 \%$ by node failure, but other MMSPEED, SAR and MGR reduce the performance maximum up to $40 \%$.

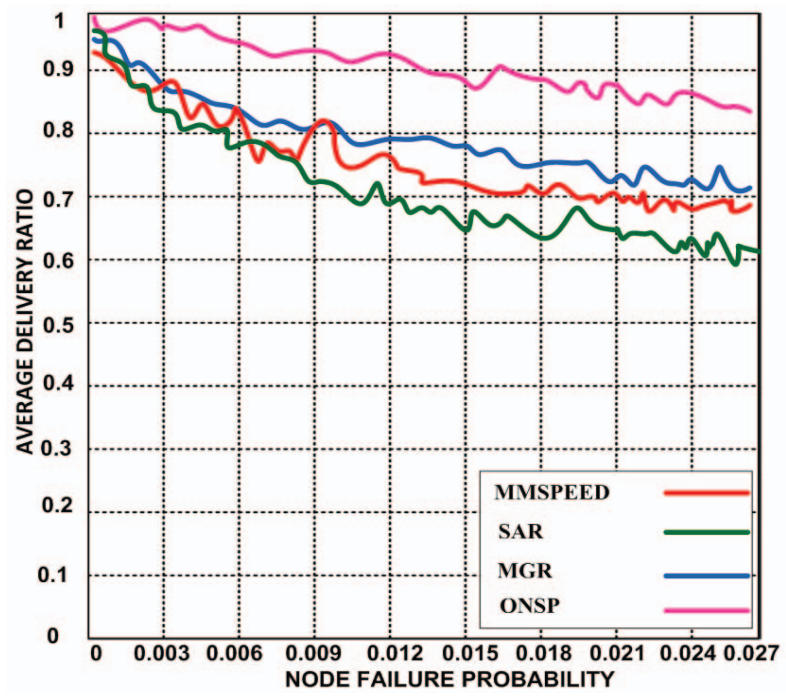

Figure 2. Average delivery rate on variable node failure probability

\section{B. Average energy consumption}

Figure 3 shows the result of energy consumption based on node failure probability. We note that ONSP outperforms MMSPEED, SAR and MGR. The energy consumption is also not highly affected due to QoS provisioning (throughput and delay). Hence, trade-off between reducing the energy consumption and improving QoS provisioning is proved that reduce the expenditure. The maximum average energy consumption for ONSP on 0.027 node failure probability is 0.037 joule/packet as compared with other protocols that range from 0.052 to 0.063 Joule/packet. The result demonstrates that ONSP consumes almost half of energy as compared to MMSPEED, SAR, and MGR due to node failure probability.

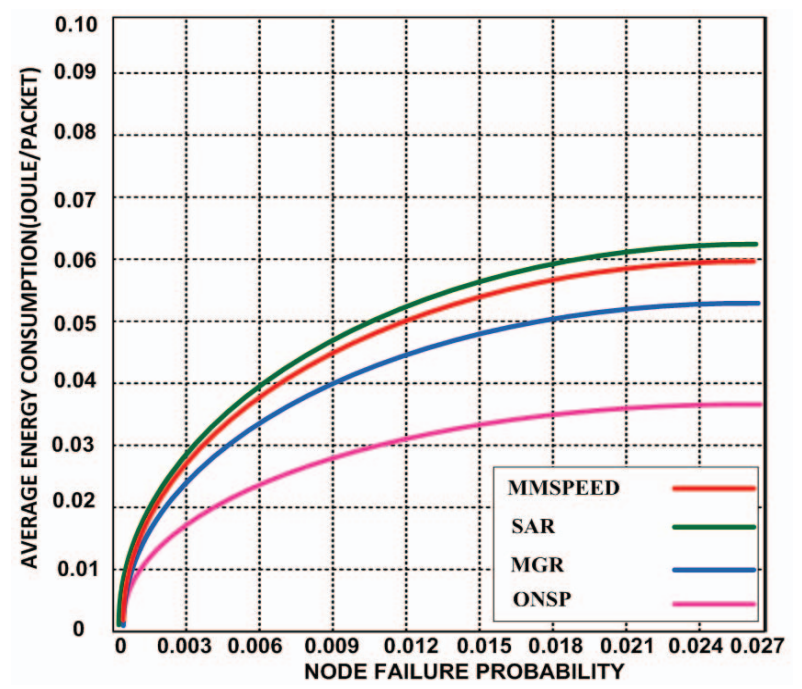

Figure 3.Average energy consumption VS node failure probability

\section{End-to-end delay}

End-to-end delay is another significant parameter for investigating the QoS based routing protocols. The packet end-to-end delay increases as time interval increases as depicted in Figure 4. In this experiment, we use variable size of packet arrival rate at the sender side. We measure an endto-end delay for both non-real time and real time data traffic. Based on the results, we validate that ONSP outperforms other participating routing protocols. The maximum end-to-end delay at the end of simulation for ONSP is 0.047 second that is almost $50 \%$ less than other routing protocols.

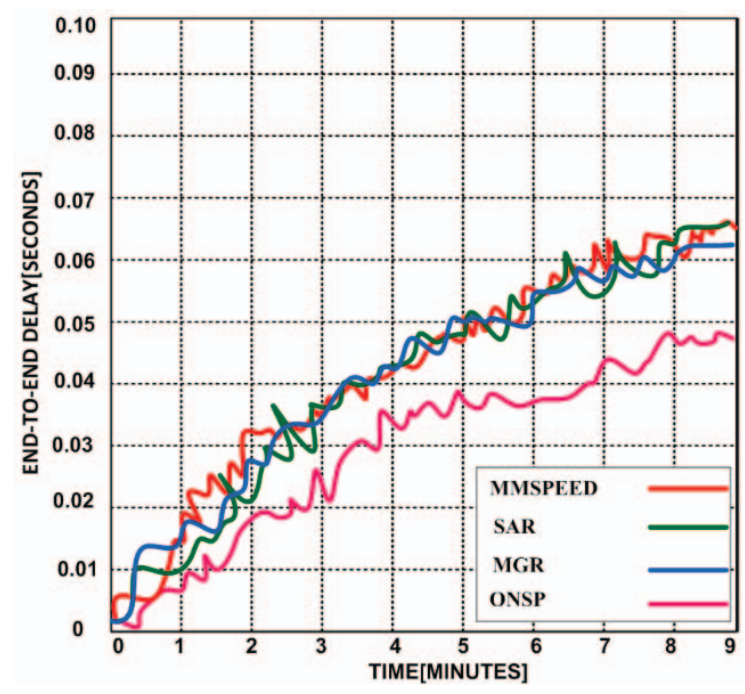

Figure 4. End-to-end delay at different time interval 


\section{Lifetime}

The main goal is to improve the lifetime of WMSN that is trade-off between energy consumption and network lifetime. We use variable network topology size to determine the lifetime of the network illustrated in Figure 5. In the experiment, we have proved that the lifetime of the network is improved using ONSP. In addition, we have also determined that increase in network size also improves the lifetime of network. The overall performance of ONSP is better than all competing routing protocols at variable network size. ONSP improves the network lifetime approximately by $37.5 \%$ which is a much better outcome.

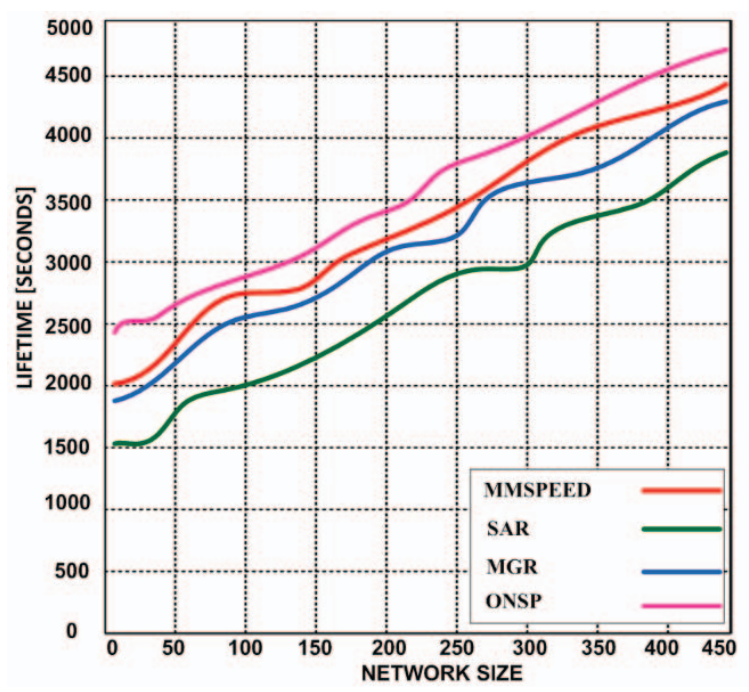

Figure 5. Lifetime of network at varying network topologies

\section{CONCLUSION}

In this paper, we have introduced the Optimized Node Selection Process (ONSP) for improving the quality of service provisioning based on multi-path routing for wireless multimedia sensor networks. This approach is designed particularly for real-time and non-real time traffic. Our approach uses the multi-path paradigm based on optimized and braided paths for improving the network life. This approach uses the optimized node process model for determining the improved node that helps route discovery. Our ONSP uses the residual energy, and received signal strength indicator to determine the next optimized node for the paths building phase.

This paper also introduces the load-balancing algorithm that helps balance the loads over all the paths in order to improve the network lifetime and guarantee the QoS provisioning. To demonstrate the strength of the proposed approach, we have used ns2. Based on simulation result, we have studied and evaluated the QoS metrics; end-to-end delay, energy consumption, network lifetime and data delivery rate. The results validate that our approach outperforms to MMSPEED,
SAR and MGR routing protocols. It is also validated that our approach can be better choice for airport surveillance system because of the extended network lifetime. In the future, we will enhance our approach by incorporating other models to obtain more outcomes for several wireless multimedia sensor network applications.

\section{REFERENCES}

[1] M. Fonoage, M. Cardei, and A. Ambrose, "A QoS based routing protocol for wireless sensor networks," in Performance Computing and Communications Conference (IPCCC), 2010 IEEE 29th International, 2010, pp. 122-129.

[2] R. Sumathi and M. Srinivas, "A Survey of QoS Based Routing Protocols for Wireless Sensor Networks," JIPS, vol. 8, pp. 589-602, 2012.

[3] K. Akkaya and M. Younis, "Energy and QoS aware routing in wireless sensor networks," Cluster computing, vol. 8, pp. 179-188, 2005.

[4] B. Bhuyan, H. K. D. Sarma, N. Sarma, A. Kar, and R. Mall, "Quality of service (QoS) provisions in wireless sensor networks and related challenges," Wireless Sensor Network, vol. 2, p. 861, 2010.

[5] L. J. García Villalba, A. L. Sandoval Orozco, A. Triviño Cabrera, and C. J. Barenco Abbas, "Routing protocols in wireless sensor networks," Sensors, vol. 9, pp. 8399-8421, 2009.

[6] S. A. Nikolidakis, D. Kandris, D. D. Vergados, and C. Douligeris, "Energy efficient routing in wireless sensor networks through balanced clustering," Algorithms, vol. 6, pp. 29-42, 2013.

S. Nikoletseas and P. G. Spirakis, "Probabilistic distributed algorithms for energy efficient routing and tracking in wireless sensor networks," Algorithms, vol. 2, pp. 121-157, 2009.

[8] M. Saleem, I. Ullah, and M. Farooq, "BeeSensor: An energy-efficient and scalable routing protocol for wireless sensor networks," Information Sciences, vol. 200, pp. 3856, 2012.

[9] A. Alanazi and K. Elleithy, "Real-Time QoS Routing Protocols in Wireless Multimedia Sensor Networks: Study and Analysis," Sensors, vol. 15, pp. 22209-22233, 2015.

[10] N. Magaia, N. Horta, R. Neves, P. R. Pereira, and M. Correia, "A multi-objective routing algorithm for Wireless Multimedia Sensor Networks," Applied Soft Computing, vol. 30, pp. 104-112, 2015.

[11] A. Martirosyan, A. Boukerche, and R. W. N. Pazzi, "Energy-aware and quality of service-based routing in wireless sensor networks and vehicular ad hoc networks,"annals of telecommunications-annales des télécommunications, vol. 63, pp. 669-681, 2008.

[12] P. Spachos, D. Toumpakaris, and D. Hatzinakos, "QoS and energy-aware dynamic routing in Wireless Multimedia Sensor Networks," in Communications (ICC), 2015 IEEE International Conference on, 2015, pp. 6935-6940.

[13] J. Park, M. Jo, D. Seong, and J. Yoo, "Disjointed Multipath Routing for Real-Time Data in Wireless Multimedia Sensor Networks," International Journal of Distributed Sensor Networks, vol. 2014, p. 8, 2014.

[14] T. Issariyakul and E. Hossain, Introduction to network simulator NS2: Springer, 2011. 